\title{
Accommodation of NLOS for Ultra-Wideband TDOA Localization in Single- and Multi-Robot Systems
}

\author{
Amanda Prorok*, Phillip Tomé ${ }^{\dagger}$, and Alcherio Martinoli* \\ * Distributed Intelligent Systems and Algorithms Laboratory, School of Architecture, Civil and Environmental Engineering \\ $\dagger$ Electronics and Signal Processing Laboratory, School of Engineering \\ Ecole Polytechnique Fédérale de Lausanne, Switzerland. Email: firstname.lastname@epfl.ch
}

\begin{abstract}
Ultra-wideband (UWB) localization is one of the most promising indoor localization methods. Yet, non-line-ofsight (NLOS) positioning scenarios can potentially cause significant localization errors and remain a challenge. In this work, we propose a novel, probabilistic UWB TDOA error model which explicitly takes into account NLOS. In order to validate our approach systematically in a real world setup, we leverage the utility of a group of mobile robots, and introduce our error model into a real-time localization framework run onboard the robots. We subsequently extend our framework by employing a collaborative localization strategy which enables the sharing of inter-robot, relative position observations. Our experimental results show how the novel TDOA error model is able to improve localization performance when information on the LOS/NLOS path condition is available. These results are complemented by additional experiments which show how a collaborative team of robots is able to significantly improve localization performance when no information on the LOS/NLOS path condition is available.
\end{abstract}

Index Terms-Non-line-of-sight, ultra-wideband, mobile robots, collaborative localization

\section{INTRODUCTION}

Accurate indoor localization is an enabling technology, with applications ranging from asset management and inventory tracking to assembly control for a variety of different industries. Within the research community, the mobile robotics domain plays an important role with a vast and continuously growing body of contributions. Popular localization sensors employed on-board robots include cameras [18], ultra-sound sensors [6], laser range finders [19] and even infrared sensors [1], and are used independently or in combination with fixed landmark beacons [2]. Although such systems have proven accurate and efficient, their great disadvantage lies in the requirement for line-of-sight (LOS). Wireless localization systems alleviate the LOS constraint, in particular those relying on UWB technology because of their large frequency spectrum, and thus enable localization over large ranges and in dynamic environments [7]. Nevertheless, they simultaneously entail issues induced by the propagation through and reflection off obstacles, which need to be addressed in order to guarantee reliable localization.

In this paper, we consider the problem of absolute localization of a team of mobile robots for unknown initial

This work was sponsored by the National Center of Competence in Research on Mobile Information and Communication Systems under grant number 51NF40-111400 of the Swiss National Science Foundation.

978-1-4577-1804-5/11/\$26.00 ㄷ 2011 IEEE

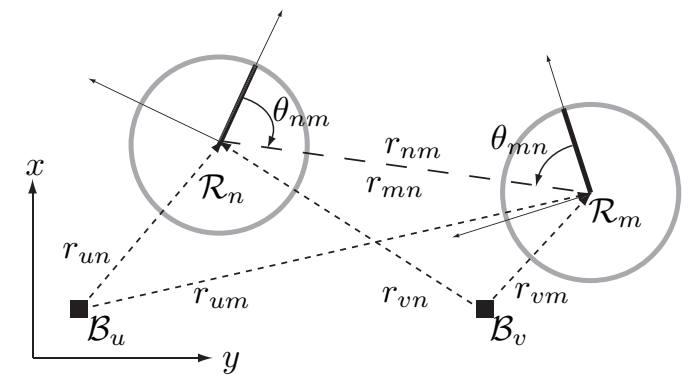

Fig. 1. System of two robots $\left(\mathcal{R}_{n}\right.$ and $\left.\mathcal{R}_{m}\right)$ and two UWB base-stations $\mathcal{B}_{u}$ and $\mathcal{B}_{v}$. The figure illustrates the robots' relative range $\left(r_{n m}\right.$ and $\left.r_{m n}\right)$ and bearing $\left(\theta_{n m}\right.$ and $\left.\theta_{m n}\right)$ values. The ranges from the base-stations to the individual robots are shown $\left(r_{u n}, r_{u m}, r_{v n}\right.$ and $\left.r_{v m}\right)$.

pose estimates. We design an algorithm targeting miniaturized, computationally limited platforms equipped with noisy, lowpower sensing modalities, and ultimately envision our solution's portability onto much smaller devices such as portable tags. Given its efficiency in solving localization problems for unknown initial conditions, and for accommodating arbitrary probability density functions, our method of choice is the particle filter, building on the probabilistic framework of MonteCarlo Localization (MCL) presented in [3]. Our localization strategy uses time-difference-of-arrival (TDOA) measurements from one or several pairs of UWB base-stations, and onboard dead reckoning information. Finally, as it is commonly known that multi-robot collaboration is able to compensate for deficiencies in the data owned by a single robot [3, 9], we extend our approach to include relative (inter-robot) range and bearing observations. We conclude our work by showing experimental results of the localization performance for both a single- and a multi-robot scenario.

\section{A. Related Work}

UWB has shown to be amongst the most promising localization techniques for indoor environments [7]. As a consequence, it has very recently been adopted by the robotics community. In [17], an UWB receiver is mounted on a mobile robot which uses a TDOA algorithm between pairs of anchor nodes to estimate its own position. The robot's self-localization algorithm is based on UWB measurements, yet it does not employ an UWB error model. The studies in [4] and [5] develop probabilistic models for biased UWB range measurements which are combined with on-board odometry data. Yet, both papers model NLOS biases within augmented-state particle filters that 
do not take LOS/NLOS path conditions and bias probability distributions into account explicitly, and are therefore limited by this simplified approach. Lastly, given the novelty of UWB positioning systems in the robotics community, to the best of our knowledge, no significant studies have been performed on the fusion of UWB with on-board exteroceptive sensors, in the case of single-robot systems, nor any on-board relative positioning sensors, in the case of multi-robot systems.

\section{B. Problem Formulation}

Our problem is illustrated in Figure 1 and described as follows. We have a multi-robot system of $N$ robots $\mathcal{R}_{1}, \mathcal{R}_{2}, \ldots \mathcal{R}_{N}$, where the number $N$ does not need to be known by the robots. The robots navigate in bounded space and are equipped with a dead-reckoning self-localization module (e.g., wheel-based odometry). For a robot $\mathcal{R}_{n}$, at time $t$, the pose $\mathbf{x}_{n, t}$ is given by the Cartesian coordinates $x_{n, t}, y_{n, t}$ and orientation $\phi_{n, t}$. At time $t$, any robot $\mathcal{R}_{n}$ in the system may emit an UWB signal and subsequently receive a TDOA value $\hat{\tau}_{u v, n, t}$ corresponding to its position $\mathbf{x}_{n, t}$ with respect to a pair of base-stations $\left\langle\mathcal{B}_{u}, \mathcal{B}_{v}\right\rangle$, each of which is fixed and well-localized in the absolute coordinate system. We denote by $\mathcal{T}_{n, t}=\left\{\left\langle\hat{\tau}_{u v, n, t}, \mathcal{B}_{u}, \mathcal{B}_{v}\right\rangle\right\}$ the set of all TDOA measurements received by robot $\mathcal{R}_{n}$ at time $t$. Hence, the TDOA measurement error is defined as the difference between the true TDOA value $\tau_{u v, n}$ and measured TDOA value $\hat{\tau}_{u v, n}$

$$
\Delta \hat{\tau}_{u v, n}=\hat{\tau}_{u v, n}-\tau_{u v, n} .
$$

The first goal of this work is to develop a TDOA measurement model $p_{u v, n}\left(\Delta \hat{\tau}_{u v, n}\right)$, which can be employed locally on each robot for localization.

Furthermore, at time $t$, a robot $\mathcal{R}_{m}$ is in the set of neighbors $\mathcal{N}_{n, t}$ of robot $\mathcal{R}_{n}$ if robot $\mathcal{R}_{m}$ can determine a range $r_{m n, t}$ and bearing $\theta_{m n, t}$ to robot $\mathcal{R}_{n}$, by means of a relative positioning module. We make the assumption that a robot $\mathcal{R}_{m}$ can communicate with a robot $\mathcal{R}_{n}$, if $\mathcal{R}_{m} \in \mathcal{N}_{n, t}$. Hence, the second goal of this work is to employ relative positioning in addition to the UWB TDOA measurements to improve the robots' localization accuracy.

\section{Experimental Setup}

Our experimental setup consists of 3 main elements (i) an UWB positioning system composed of four base-stations, (ii) a group of four mobile robots, and (iii) an overheadcamera serving as ground truth positioning system. The UWB localization system employed in this work is commercially available from Ubisense ${ }^{1}$, Series 7000 (sensors and compact tags). It is installed on the ceiling of a $40 \mathrm{~m}^{2}$ laboratory, with each of the four base-stations mounted at the extremities of a $9 m^{2}$ square robotic arena as depicted in Figure 2. The robots are all equipped with relative positioning modules, as well as narrowband communication modules which, in case of collaboration, enable them to share data as detailed in Section I-B, above. Finally, the overhead camera system runs

\footnotetext{
${ }^{1}$ http://www.ubisense.net
}

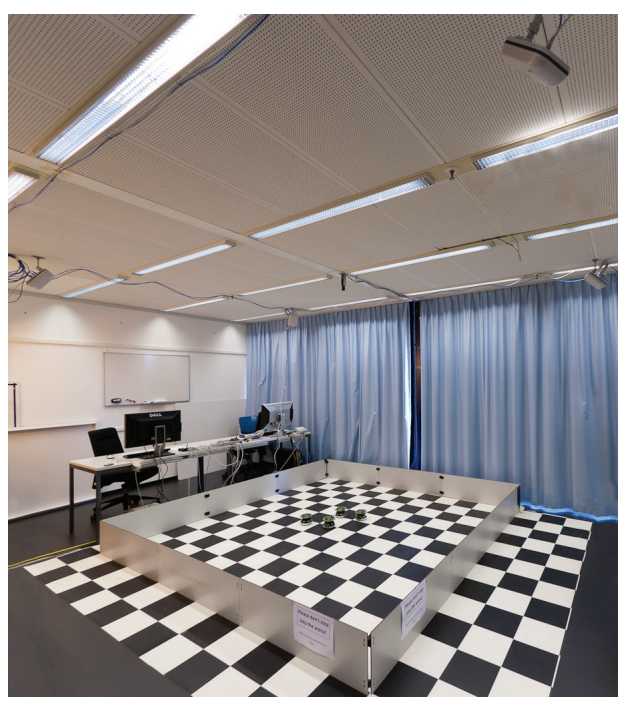

Fig. 2. Experimental setup in the laboratory space. The UWB base-stations are mounted on the ceiling above the extremities of a $9 \mathrm{~m}^{2}$ robotic arena, and an overhead camera is mounted on the ceiling above the center of the arena.

on a central processor which also enacts the synchronization of available ground truth positioning data with all incoming raw sensor data (from the UWB system as well as from the robots), enabling offline performance evaluation. For further elaboration and technical details, the reader should refer to the experiments' section (Section V).

\section{Monte-Carlo Localization}

In this section, we briefly review Monte-Carlo Localization (MCL) [20], as it forms the baseline for our localization algorithm. We first elaborate the single-robot MCL algorithm, and then, in Section II-B, extend this standard MCL formalism to a decentralized, collaborative adaptation (the reader should also refer to [12] for further details).

\section{A. Single-robot $M C L$}

MCL, as many other localization techniques, relies on the formalism of the Bayes filter. The Bayes filter is a recursive algorithm which estimates the probability density of a posterior state, conditioned on the measurements collected. Let us from hereon consider a robot $\mathcal{R}_{n}$. At time $t$, after a sequence of motion control actions $u_{n, t}$ and a sequence of observations $z_{n, t}$ the filter is denoted

$$
\begin{aligned}
\operatorname{Bel}\left(\mathbf{x}_{n, t}\right)=\eta p\left(z_{n, t} \mid \mathbf{x}_{n, t}\right) \int & p\left(\mathbf{x}_{n, t} \mid \mathbf{x}_{n, t-1}, u_{n, t-1}\right) \\
& \operatorname{Bel}\left(\mathbf{x}_{n, t-1}\right) \mathbf{d} \mathbf{x}_{n, t-1}
\end{aligned}
$$

where $\operatorname{Bel}\left(\mathbf{x}_{n, t}\right)$ estimates the posterior of state $\mathbf{x}_{n, t}$ and is called a belief. The value $\eta$ is a normalization constant, $p\left(z_{n, t} \mid \mathbf{x}_{n, t}\right)$ is a measurement model, and $p\left(\mathbf{x}_{n, t} \mid \mathbf{x}_{n, t-1}, u_{n, t-1}\right)$ a motion model.

The main idea of MCL lies in the way the belief is represented-samples, or particles, are drawn from the posterior probability distribution of the robot pose to form a set of particles. By weighting these particles one obtains a discrete 
probability function that approximates the continuous belief $\operatorname{Bel}\left(\mathbf{x}_{n, t}\right)$, and hence we have

$$
\operatorname{Bel}\left(\mathbf{x}_{n, t}\right) \sim\left\{\left\langle\mathbf{x}_{n, t}^{[i]}, w_{n, t}^{[i]}\right\rangle \mid i=1, \ldots, M\right\}=X_{n, t}
$$

where $M$ is the number of particles, $\mathbf{x}_{n, t}^{[i]}$ is a sample of the random variable $\mathbf{x}_{n, t}$ (the pose), and $w_{n, t}^{[i]}$ is its weight. The symbol $X_{n, t}$ refers to the set of particles $\left\langle\mathbf{x}_{n, t}^{[i]}, w_{n, t}^{[i]}\right\rangle$ at time $t$ belonging to robot $\mathcal{R}_{n}$. In contrast to other methods (for example Kalman filtering), the advantage of this form of representation is that it can approximate probability densities of any shape. Given this flexibility, MCL is, thus, able to accommodate arbitrary sensor characteristics and noise distributions. Also, by tuning the total number of particles used, MCL can adapt to variable computational capacities, at the cost of accuracy in the reproduction of the targeted continuous probability densities.

At the beginning of a localization exercise, the set of particles $X_{n, 0}$ is initialized: if the initial pose is known, the particles are drawn from a probability distribution (e.g., a Normal distribution) centered around the given pose, and if the initial pose is unknown, the particles are drawn from a uniform distribution covering the whole environment. Then, in order to estimate the robot's pose at each time step, a recursive update is made. These steps, when performed for the whole set of $M$ particles, can be formulated as a recursive update algorithm (as presented in [20]), for a given robot $\mathcal{R}_{n}$.

For the sake of completeness, this routine is shown here in Algorithm 1. Line 3 shows the application of the motion model, where $u_{n, t}$ represents dead-reckoning information-in our case, odometry readings. Line 4 shows the application of the measurement model where $\mathcal{T}_{n, t}$ represents the TDOA data. This TDOA measurement model lies at the core of our current work, and is elaborated in greater detail in Section III.

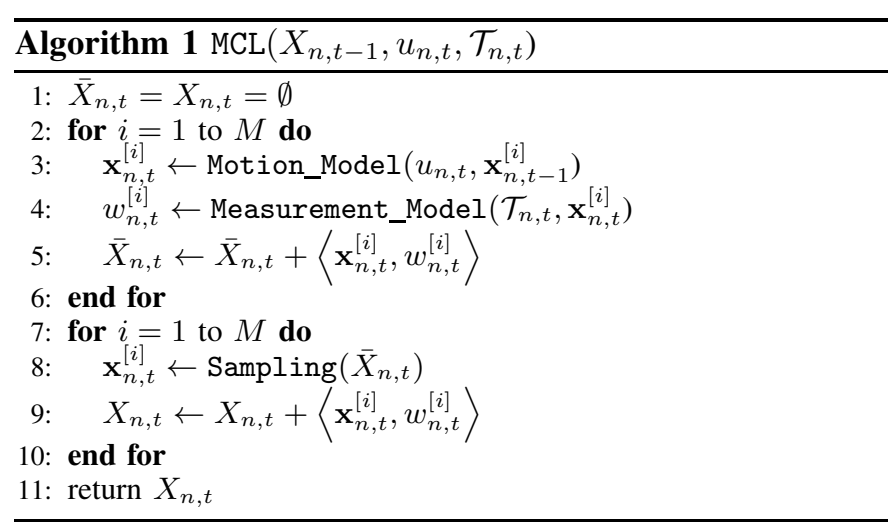

\section{B. Multi-robot $M C L$}

The framework presented above takes into account a single robot. However, when operating a collaborative multi-robot system, the baseline formalism must be adapted to integrate measurements taken on different platforms [3]. Motivated by the goal of overcoming the limitations of current multi-robot localization algorithms, which to date are hard to employ on
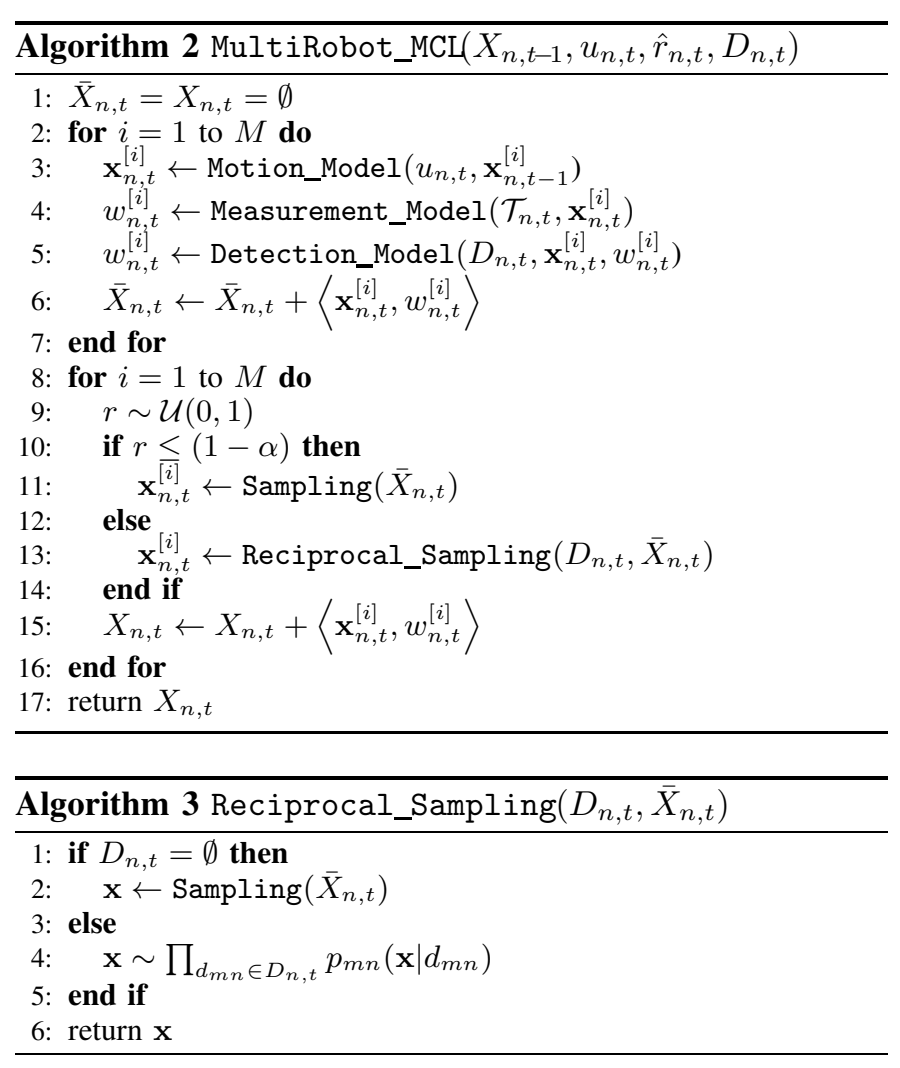

large-scale, distributed systems for unknown initial conditions, we developed an any-time, fully scalable collaborative localization algorithm. This algorithm additionally takes advantage of reciprocal robot observations to reduce the number of particles needed to localize, thus reducing the computational overhead, which is a crucial factor for resource-bounded mobile platforms.

If we make the assumption that individual robot poses are independent, we can formulate the event that robot $\mathcal{R}_{n}$ is detected by robot $\mathcal{R}_{m}$ as

$$
\begin{aligned}
& \operatorname{Bel}\left(\mathbf{x}_{n, t}\right)=p\left(\mathbf{x}_{n, t} \mid z_{n, 0 . . t}, u_{n, 0 . . t}\right) \\
& \quad \int p\left(\mathbf{x}_{n, t} \mid \mathbf{x}_{m, t}, r_{m n, t}, \theta_{m n, t}\right) \operatorname{Bel}\left(\mathbf{x}_{m, t}\right) \mathrm{d} \mathbf{x}_{m, t}
\end{aligned}
$$

where $p\left(\mathbf{x}_{n, t} \mid z_{n, 0 . . t}, u_{n, 0 . . t}\right)$ describes the $n^{\text {th }}$ robot's current belief, and $\int p\left(\mathbf{x}_{n, t} \mid \mathbf{x}_{m, t}, r_{m n, t}, \theta_{m n, t}\right) \operatorname{Bel}\left(\mathbf{x}_{m, t}\right) \mathrm{d} \mathbf{x}_{m, t}$ describes the $m^{\text {th }}$ robot's belief about the position of robot $\mathcal{R}_{n}$. For such a collaboration to take place, robot $\mathcal{R}_{m}$ needs to communicate $r_{m n, t}, \theta_{m n, t}$ and $\operatorname{Bel}\left(\mathbf{x}_{m, t}\right)$ to robot $\mathcal{R}_{n}$. Thus a communication message is composed as $d_{m n, t}=\left\langle r_{m n, t}, \theta_{m n, t}, X_{m, t}\right\rangle$. If several robots in a neighborhood $\mathcal{N}_{n, t}$ communicate with robot $\mathcal{R}_{n}$, the received information is the set of all communication messages $D_{n, t}=$ $\left\{d_{m n, t} \mid \mathcal{R}_{m} \in \mathcal{N}_{n, t}\right\}$. We note that the collaborative aspect of this formalism lies in the integration of robot $\mathcal{R}_{m}$ 's belief into that of robot $\mathcal{R}_{n}$. This update step is shown in Algorithm 2 (line 5).

In addition to using a robot detection model for updating the belief representation $\operatorname{Bel}\left(\mathbf{x}_{n, t}\right)$, our collaborative approach 
relies on a reciprocal sampling method, which alleviates issues (such as particle collapse or particle depletion) due to finite particle set sizes. As for a standard MCL algorithm, the posterior estimate of reciprocal MCL is represented by $\operatorname{Bel}\left(\mathbf{x}_{n, t}\right)$ the difference between the two methods lies in the proposal distribution. Let us refer to the iterative process described in Algorithm 2: instead of sampling from $\operatorname{Bel}\left(\mathbf{x}_{n, t-1}\right)$ in line 11 , the reciprocal MCL algorithm samples from the distribution $\mathbf{x}_{n, t}^{[i]} \sim p\left(D_{n, t} \mid \mathbf{x}_{n, t}^{[i]}\right)$, according to a robot detection model (line 13). Thus, samples are drawn at poses which are probable given the reciprocal robot observations, and which are independent of the previous belief $\operatorname{Bel}\left(\mathbf{x}_{n, t-1}\right)$. Then, by employing the reciprocal sampling algorithm within the collaborative paradigm of our general framework, a detected robot augments its own belief with new pose estimates deduced from reciprocal robot observations with a fixed proportion of $\alpha$. In particular, as this method exploits the information available in a whole robot team, it continuously creates particles in areas of the pose space which are likely to be significant, and allows for very small particle set sizes.

The reciprocal sampling routine is shown in Algorithm 3, where line 4 represents the reciprocal sampling step. Algorithm 2 shows the complete routine of multi-robot reciprocal MCL. The second part (lines 8-16) resamples particles from the weighted proposal distribution to create a new, updated pose belief. Particles are sampled from the robot's own belief with a probability $1-\alpha$, and with a probability of $\alpha$, particles are sampled from the probability density function proposed by the detection model (line 13). Without going into further details in this paper, we performed empirical tests which showed that small proportions of $\alpha$ were beneficial, and thus a proportion of $\alpha=0.05$ was employed throughout our work. Further, there are a multitude of methods which can be applied to sample from a given distribution. Here, we employ the slice sampling method [11], which is a low-cost method based on Markov chains, and particularly useful since it can sample from arbitrary shaped distributions. The symbol $\mathcal{U}$ on line 9 of Algorithm 2 refers to the uniform distribution.

\section{UWB TDOA MEASUREMENT MODEL}

UWB is a radio technology which is characterized by its very large bandwidth compared to conventional narrowband systems, and in particular features high positioning accuracy (due to a high time resolution) and high material penetrability (due to the large bandwidth). Despite these desirable traits, the resolution of multipath signals leads to complex TOA algorithms prone to estimation errors, which inevitably leads to ranging inaccuracies. In this section, we first develop a baseline error model for TOA measurements, and then extend it analogously to model the errors of TDOA measurements. We note that as of the following, the terms TOA and TDOA are used interchangeably with the terms range and range difference, as they differ only by a constant factor (propagation speed). For clarity, we omit the subscript $t$ in the following derivations.

\section{A. Preliminaries: TOA Measurement Model}

We employ a popular error model [16] for the range between a base-station $\mathcal{B}_{u}$ and a target node $\mathcal{R}_{n}$

$$
\hat{r}_{u n}=r_{u n}+\epsilon_{u n}+Y b_{u n}
$$

where $r_{u n}$ represents the true distance, $b_{u n}$ is a non-negative distance bias introduced by a NLOS signal propagation, and $\epsilon_{\text {un }} \sim \mathcal{N}\left(0, \sigma_{\mathcal{N}}^{2}\right)$ is a zero-mean Gaussian measurement noise with variance $\sigma_{\mathcal{N}}^{2}$, common to all base-stations. The random variable $Y$ follows a Bernoulli distribution, i.e., it takes the value 1 with probability $\left(1-\mathrm{P}_{L_{u n}}\right)$ and the value 0 with probability $\mathrm{P}_{L_{u n}}$, where $\mathrm{P}_{L_{u n}}$ is the probability of measuring a LOS path, and correspondingly, $\left(1-\mathrm{P}_{L_{u n}}\right)$ is the probability of measuring a NLOS path. Whereas modeling $\epsilon_{u n}$ is straightforward, modeling the bias $b_{u n}$ is less obvious. Current work discusses a variety of viable statistical models with exponential behavior [10,15]. Indeed, biases may not only be caused by multipath propagation, but also by signal delay or by signal attenuation, and thus are dependent on bandwidth and distance. Despite the complexity of NLOS error patterns, we resort to a statistical model, the log-normal distribution, as it is shown to best characterize the spatial NLOS error behavior in the comprehensive measurement campaign of [10]. Thus, the bias $b_{u n}$ follows a log-normal distribution $b_{u n} \sim \ln \mathcal{N}\left(\mu_{\operatorname{ln\mathcal {N}}, u}, \sigma_{\operatorname{ln\mathcal {N}}, u}\right)$, and is unique for each basestation $\mathcal{B}_{u}$.

Our TOA measurement model returns the likelihood that a given range error occurs. For a range error defined as

$$
\Delta \hat{r}_{u n}=\hat{r}_{u n}-r_{u n}
$$

the TOA measurement model describes the likelihood of $\Delta \hat{r}_{u n}$ occurring when a robot $\mathcal{R}_{n}$ measures a certain range distance $\hat{r}_{u n}$ from a base-station $\mathcal{B}_{u}$ at an actual position $\mathbf{x}_{n}$ with a nominal range $r_{u n}$ from a base-station $\mathcal{B}_{u}$. In order to differentiate the two path conditions, we denote the event of a LOS path from location $\mathbf{x}_{n}$ to base-station $\mathcal{B}_{u}$ as $L_{u n}$, and the event of a NLOS path $\bar{L}_{u n}$, respectively. Then, in order to model the range error, we describe the probability distribution of $\Delta \hat{r}_{u n}$ as the sum of the random variables $b_{u n}$ and $\epsilon_{u n}$, drawn from their respective probability distributions. For a lognormal probability density function $p_{\operatorname{ln\mathcal {N}}, u}(b)$ with parameters $\mu_{\operatorname{ln\mathcal {N}}, u}$ and $\sigma_{\operatorname{ln\mathcal {N}}, u}$, and a normal probability density function $p_{\mathcal{N}}(\epsilon)$ with a standard deviation $\sigma_{\mathcal{N}}$, the probability density of an error $\Delta \hat{r}_{u n}$, occurring in a NLOS condition, can be written as

$$
p_{u n}\left(\Delta \hat{r}_{u n} \mid \bar{L}_{u n}\right)=\left(p_{\operatorname{ln\mathcal {N}}, u} * p_{\mathcal{N}}\right)\left(\Delta \hat{r}_{u n}\right)
$$

which is the convolution of the probability density function of the bias value, with the probability density function of the Gaussian noise value. Correspondingly, we can write the probability density of an error $\Delta \hat{r}_{u n}$, occurring in a LOS condition, as

$$
p_{u n}\left(\Delta \hat{r}_{u n} \mid L_{u n}\right)=p_{\mathcal{N}}\left(\Delta \hat{r}_{u n}\right)
$$

Finally, with use of the total probability theorem, we combine the above equations to obtain the probability density of $\Delta \hat{r}_{u n}$ 


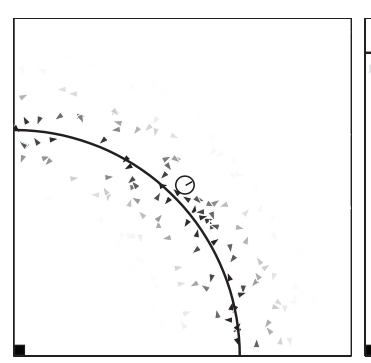

(a)

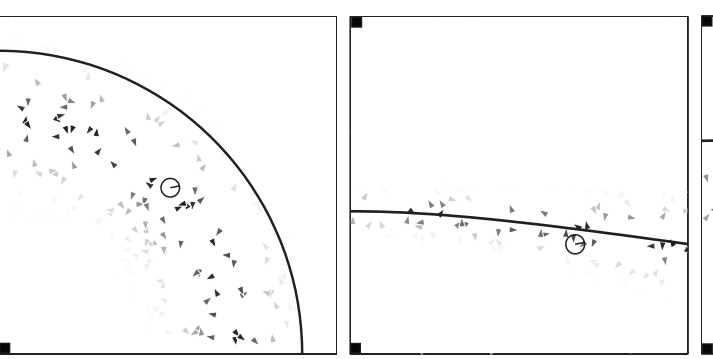

(b) (c)

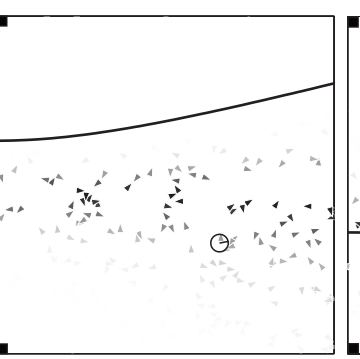

(d)

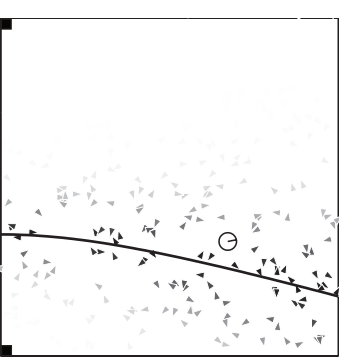

(e)

Fig. 3. Illustration of TOA and TDOA measurement models within a particle filter. The pose estimates (particles) are represented by triangles with increasing transparency for decreasing weights. The curved lines represent the noisy/biased TOA range measurements in (a)-(b) and noisy/biased TDOA measurements in (c)-(e). The robot body shows the actual robot position. The TOA measurement model for a single base-station is illustrated in (a) for a LOS condition and (b) for a NLOS condition. The TDOA measurement model for a single base-station pair is shown in (c) for both base-stations in LOS conditions, (d) for the bottom base-station in NLOS, the top base-station in LOS conditions, and (e) for both base-stations in NLOS conditions.

(which is obtained by measuring a range $\hat{r}_{u n}$ at an actual, nominal range $r_{u n}$ ) as

$$
\begin{aligned}
p_{u n}\left(\Delta \hat{r}_{u n}\right) & =p_{u n}\left(\Delta \hat{r}_{u n} \mid L_{u n}\right) \cdot \mathrm{P}_{L_{u n}} \\
& +p_{u n}\left(\Delta \hat{r}_{u n} \mid \bar{L}_{u n}\right) \cdot\left(1-\mathrm{P}_{L_{u n}}\right) .
\end{aligned}
$$

Figures 3 (a) and (b) show an application of Equation 9, weighting particles in (a) a LOS scenario and (b) a NLOS scenario, for a single base-station, where the probability of $\mathrm{P}_{L_{u n}}$ is assumed known (in this case we have (a) $\mathrm{P}_{L_{u n}}=1$ and (b) $\mathrm{P}_{L_{u n}}=0$ ). We observe that the model produces particles with high weights in the area of the actual robot position, even when the measurement is biased. Also, it is clear from the figures that information on the actual LOS/NLOS path condition is essential to obtain good localization.

\section{B. TDOA Measurement Model}

In practice, TOA systems are rarely implemented due to the complexity induced by the required synchronization of a mobile node with the base-stations. Instead, it is a common choice to implement TDOA systems which are significantly more practical, since only the synchronization among base-stations is required. Thus, the direct range measurement between a mobile node and base-station is replaced by the difference between two individual range measurements each taken at a different base-station.

Extending the TOA formalism shown above, we define the difference range value (i.e. TDOA) between two base-stations $\mathcal{B}_{u}$ and $\mathcal{B}_{v}$ to a target node $\mathcal{R}_{n}$ as

$$
\hat{\tau}_{u v, n}=\hat{r}_{u n}-\hat{r}_{v n}
$$

and then easily model the TDOA error $\Delta \hat{\tau}_{u v, n}$ as previously shown in Equation 1. Simultaneously, we can describe the TDOA error as the difference between the range errors occurring at the individual base-stations $\mathcal{B}_{u}$ and $\mathcal{B}_{v}$ as described in Equation 5, resulting in

$$
\Delta \hat{\tau}_{u v, n}=\Delta \hat{r}_{u n}-\Delta \hat{r}_{v n}
$$

Finally, we describe the probability density of a given TDOA measurement error $\Delta \hat{\tau}_{u v, n}$ as the substraction of two random variables drawn from the probability densities describing the
TOA error models of the two respective base-stations. We use the results of Equations 9 and 11 to model this resulting probability density as

$$
p_{u v, n}\left(\Delta \hat{\tau}_{u v, n}\right)=\left(p_{u n} * p_{v n}^{-}\right)\left(\Delta \hat{\tau}_{u v, n}\right)
$$

which is a convolution of the probabilities to measure range errors $\Delta \hat{r}_{u n}$ and $\Delta \hat{r}_{v n}$, and where we denote $p_{v n}^{-}(x)=p_{v n}(-x)$ for all $x$.

Algorithm 4 shows the particle weight update equation based on the probability density function of Equation 12 . Figures 3 (c)-(e) show an application of the TDOA error model of Equation 12, weighting particles in (c) a LOS condition for both base-stations, (d) a LOS condition for the top base-station and a NLOS condition for the bottom base-station and (e) a NLOS condition for both base-stations. We see that Equation 12 is able to capture all possible conditions. Also, we observe that the model produces particles with high weights in the area of the actual robot position. Finally, analogous to the conclusions of Figure 3(a) and (b), we note that the availability of information on the actual LOS/NLOS path condition is essential for good localization.

\begin{tabular}{l}
$\overline{\text { Algorithm } 4 \text { Measurement_Model }\left(\mathcal{T}_{n, t}, \mathbf{x}_{t}^{[i]}\right)}$ \\
\hline 1: $w \leftarrow \prod_{\left\langle\hat{\tau}_{u v, n, t}, \mathcal{B}_{u}, \mathcal{B}_{v}\right\rangle \in \mathcal{T}_{n, t}} p_{u v, n}\left(\Delta \hat{\tau}_{u v, n}\right)$ \\
2: return $w$
\end{tabular}

\section{Estimation of TDOA Model Parameters}

In order to employ accurate error models on the measured quantities during localization, we perform an a priori offline system identification process which returns a set of optimized model parameter values. In other words, based on a set of gathered data, we estimate the parameters $\mu_{\operatorname{ln\mathcal {N}}, u}, \sigma_{\operatorname{ln\mathcal {N}}, u}$ describing the log-normal bias distributions of each individual base-station $\mathcal{B}_{u}$, as well as the standard deviation of the common Gaussian noise $\sigma_{\mathcal{N}}$. Simultaneously, we estimate the proportion of measurements made in LOS path conditions $\mathrm{P}_{L_{u n}}$ for each base-station $\mathcal{B}_{u}$, at all queried positions $\mathbf{x}_{n}$ in the data set. The data used for the identification procedure was gathered in a dedicated robotic experiment, and is independent 


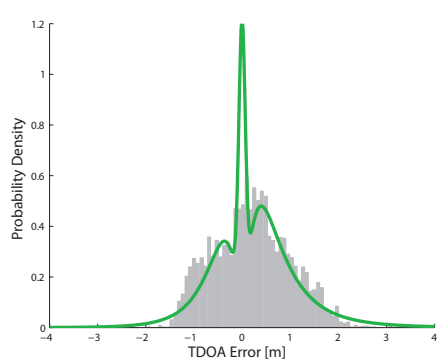

(a)

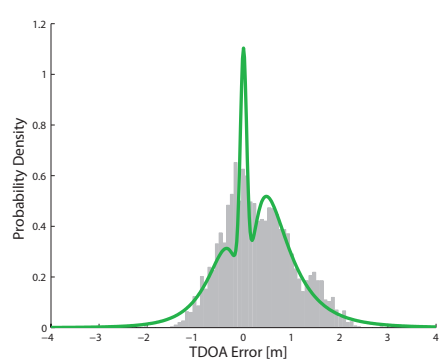

(b)

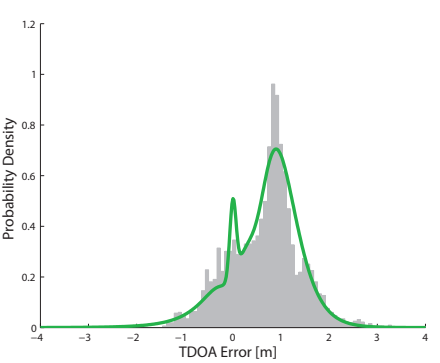

(c)

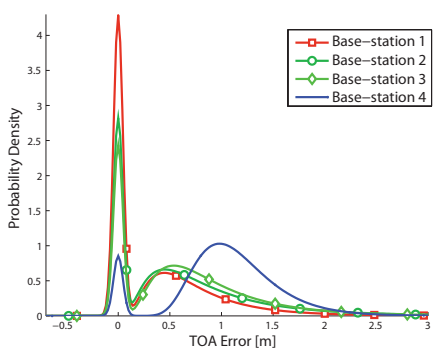

(d)

Fig. 4. The graphs show the estimated error models. Normalized histograms of the actual TDOA error data, and a superposition of the estimated probability density function of Equation 12 for base-station pairs (a) $\left\langle\mathcal{B}_{1}, \mathcal{B}_{2}\right\rangle$, (b) $\left\langle\mathcal{B}_{1}, \mathcal{B}_{3}\right\rangle$ and (c) $\left\langle\mathcal{B}_{1}, \mathcal{B}_{4}\right\rangle$. (d) Estimated probability density function of Equation 9 for all individual base-stations.

\begin{tabular}{|c||c|c|c|c|}
\hline & $\mathcal{B}_{1}$ & $\mathcal{B}_{2}$ & $\mathcal{B}_{3}$ & $\mathcal{B}_{4}$ \\
\hline \hline$\mu_{\ln \mathcal{N}}$ & -0.43 & -0.3 & -0.24 & -0.09 \\
\hline$\sigma_{\ln \mathcal{N}}$ & 0.611 & 0.7 & 0.61 & 0.33 \\
\hline$\sigma_{\mathcal{N}}$ & \multicolumn{4}{|c|}{0.047} \\
\hline $\mathrm{P}_{L}$ & 0.49 & 0.32 & 0.28 & 0.09 \\
\hline \multicolumn{5}{c}{ TABLE I }
\end{tabular}

OPTIMIZED MODEL PARAMETER VALUES PER BASE-STATION.

from the data sets used in the experiments performed later in this paper. Four robots perform a random walk in the arena for 16 minutes so that 4000 TDOA data points are gathered per base-station pair, ensuring homogeneous coverage of the arena space. By processing the UWB TDOA data and associated ground truth positions, we obtain a distribution of TDOA error values (refer to Equation 1). Then, based on our probability density model of Equation 12, we minimize the Kolmogorov-Smirnov distance with the empirical cumulative density function (CDF) obtained from the collected data. This optimization procedure is done using a numerical gradientdescent algorithm, namely the BFGS Quasi-Newton method. The average Kolmogorov-Smirnov distance obtained after minimization is 0.036 , showing a good representation of the data.

The resulting optimized parameter values (thirteen in total) are reported in Table I. Figures 4 (a)-(c) show normalized histograms of the actual TDOA error data (with base-station $\mathcal{B}_{1}$ as the reference base-station), and a superposition of the probability density function of Equation 12 with the optimized parameters for three base-station pairs. Figure 4 (d) shows the probability density function of Equation 9 with the optimized parameters for all individual base-stations.

\section{Estimation of the LOS/NLOS Path Condition}

As evident from the discussion of Figure 3, an accurate estimation of the LOS/NLOS path condition is essential for good localization. Without knowledge of the ground truth position, this can be done only in an overdetermined system (e.g., for 5 base-stations in 3D), with only one base-station in NLOS and all others in LOS. However, with the knowledge of the ground truth position and a fully determined system, we can determine the LOS/NLOS condition if we know that at least one of the base-stations is in LOS. Thus, we can resolve this condition in real-time only in very specific cases. In the experiments' section of this paper (Section V), we will discuss the implications that different assumptions on the path conditions may have on the performance of the system. It is in this context that we hypothesize the knowledge of optimal LOS/NLOS path conditions. In order to emulate this knowledge, we develop a simple algorithm which, based on the measured TDOA values and their associated ground truth positions, is able to provide an estimate of the occurring bias for each base-station.

For a given robot $\mathcal{R}_{n}$, and for all elements $\left\langle\hat{\tau}_{u v, n}, \mathcal{B}_{u}, \mathcal{B}_{v}\right\rangle$, we pose the equation (see Equations 5 and 10 )

$$
\hat{\tau}_{u v, n}=\tau_{u v, n}+b_{u n}-b_{v n}
$$

where $b_{u n}$ and $b_{v n}$ are unknowns. Note that the zero-mean Gaussian measurement noise values $\epsilon_{u n}$ and $\epsilon_{v n}$ are significantly smaller than the bias, and thus we ignore their contribution here. Still, this system of equations remains
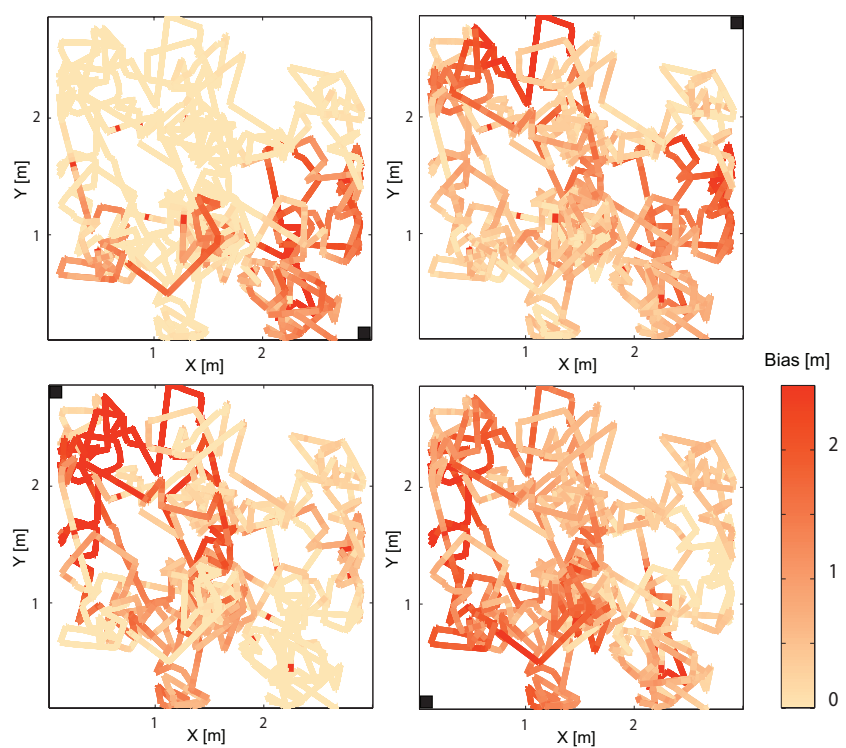

Fig. 5. The graphs show the estimated bias values for each base-station (marked in the corners), along the tracks of the mobile robot during data collection. The robot's ground truth position is continuously tracked by an overhead camera. 
underdetetrmined. Hence, we make the assumption that at least one base-station has a bias equal to zero, which is likely true in our experimental setup, but not generally true. We can now solve this linear system of equations and find all bias values $b_{u n}$ for all base-stations $\mathcal{B}_{u}$ that provided a TDOA measurement.

Figure 5 shows an example application of the estimation algorithm. A mobile robot moves randomly inside our experimental arena while ground truth tracking is performed and TDOA measurements are taken. The tracked positions shown in the plots are colored to illustrate the associated estimated bias values for each of the four base-stations in our setup. As expected, we observe that higher bias values occur close to the respective base-stations as the arena walls lie in the direct signal path (see Figure 2 for an image of the physical installation).

\section{RAnge \& BeARing Measurement Model}

As introduced in Section II-B, the robots use relative observations to collaborate. In this Section we detail our robot detection model, which is built upon relative range and bearing measurements. The idea of the range and bearing model is to propose a probability density function which is based on the relative observations made by the detection sensors, and which is also based on the belief of the detecting robot. We then simultaneously use this probability density function as an observation model in the belief update, and as a proposal distribution for the reciprocal sampling routine. Again, for clarity, we omit the subscript $t$ in the following derivations. A robot $\mathcal{R}_{m}$ detects a robot $\mathcal{R}_{n}$ with a range $r_{m n}$ and relative bearing $\theta_{m n}$. We formulate the detection model as $p_{m n}\left(\mathbf{x}_{n} \mid d_{m n}\right)$ which describes the probability that robot $\mathcal{R}_{m}$ detects robot $\mathcal{R}_{n}$ at pose $\mathbf{x}_{n}=\left[x_{n} y_{n} \phi_{n}\right]$, given the detection data $d_{m n}$. For a given particle $i$ in robot $\mathcal{R}_{m}$ 's belief, we define the range difference $\Delta r_{m n}$, and the bearing difference $\Delta \theta_{m n}$. The range and bearing differences are given by the geometric relations

$$
\begin{aligned}
\Delta r_{m n} & =\sqrt{\Delta x_{m n}^{2}+\Delta y_{m n}^{2}}-r_{m n} \\
\Delta \theta_{m n} & =\operatorname{atan} 2\left(\Delta y_{m n}, \Delta x_{m n}\right)-\left(\phi_{m}^{[i]}+\theta_{m n}\right)
\end{aligned}
$$

where we denote $\Delta x_{m n}=\left(x_{m}^{[i]}-x_{n}\right) \quad$ and $\Delta y_{m n}=\left(y_{m}^{[i]}-y_{n}\right)$. Assuming Gaussian noise and knowledge of the range and bearing standard deviation ( $\sigma_{r}$ and $\sigma_{\theta}$, respectively), and the independence of range and bearing measurements, the detection probability is

$$
\begin{aligned}
p_{m n}\left(\mathbf{x}_{n} \mid d_{m n}\right)= & \eta \cdot \sum \Phi\left(\left[\begin{array}{c}
\Delta r_{m n} \\
\Delta \theta_{m n}
\end{array}\right],\left[\begin{array}{cc}
\sigma_{r}^{2} & 0 \\
0 & \sigma_{\theta}^{2}
\end{array}\right]\right) \cdot w_{m}^{[i]} \\
& \left\langle\begin{array}{c}
\mathbf{x}_{m}^{[i]} \\
w_{m}^{[i]}
\end{array}\right\rangle \in X_{m}
\end{aligned}
$$

where $\Phi(\cdot, \Sigma)$ is the zero-mean multivariate normal probability distribution with the covariance matrix $\Sigma$ and where $\eta$ is a normalization constant. Also, in the case where robot $\mathcal{R}_{n}$ reciprocally detects robot $\mathcal{R}_{m}$, it can use the additional information of its own relative observations to determine

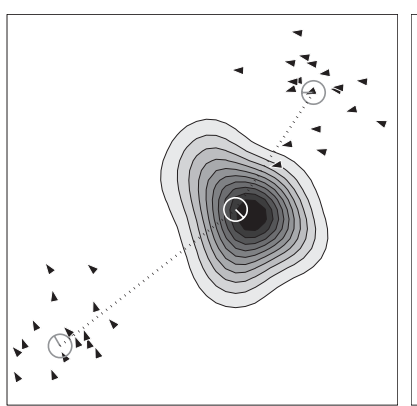

(a)

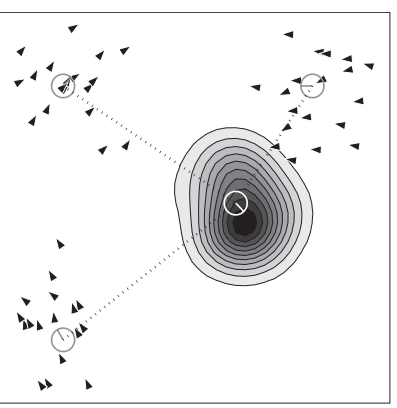

(b)
Fig. 6. Detection model for multiple detecting robots, (a) for two robots and (b) for three robots. Here, a set of 20 particles is shown, represented by oriented triangles. The detected robot is shown in white. The model's probability density is superimposed on the detected robot. The dotted line and the orientation of the robots show the actual relative range and bearing. The particle positions were generated randomly from a normal distribution ( $\sigma_{x}=\sigma_{y}=0.2 \mathrm{~m}$, and $\sigma_{\phi}=0.2 \mathrm{rad}$ ), and range values are perturbed by a white noise with $\sigma_{r}=20 \%$ and for the bearing values with $\sigma_{\theta}=0.2 \mathrm{rad}$.

the orientation difference $\Delta \phi_{m n}$, which is defined by the following geometric relation

$$
\Delta \phi_{m n}=\pi-\phi_{m}^{[i]}-\phi_{n}+\theta_{m n}-\theta_{n m} .
$$

The detection probability is then augmented by an additional component, resulting in

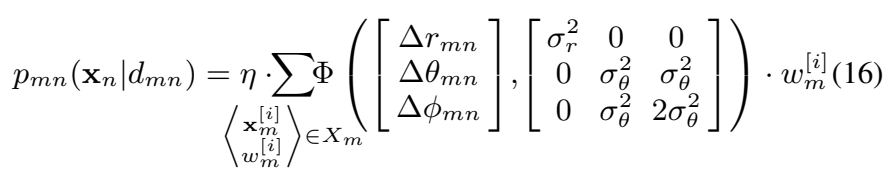

Finally, the detection model can be formulated as an update equation as shown in Algorithm 5. Algorithm 3 shows how samples are drawn from the detection model in the reciprocal sampling routine. Figure 6 shows an illustration of the probability density function resulting from the detection model, (a) for two detecting robots and (b) for three detecting robots. We observe how, for an increasing number of detecting robots, the localization accuracy increases.

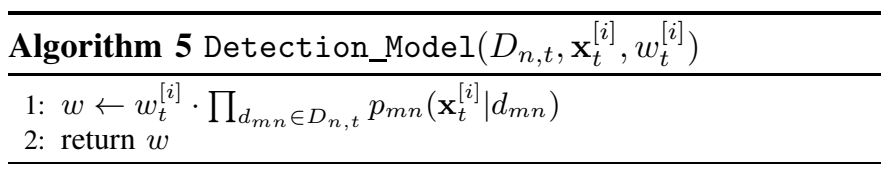

\section{EXPERIMENTS}

The framework of our experimental setup is as elaborated in Section I-C and as previously shown in Figure 2. The robots employed in our experiments are Khepera III robots, which are differential drive robots of $12 \mathrm{~cm}$ diameter produced by K-Team corporation ${ }^{2}$. The Khepera III robot has a KoreBot extension board providing a standard embedded Linux operating system on an Intel XSCALE PXA-255 processor running at $400 \mathrm{MHz}$. Communication is enabled through an IEEE $802.11 \mathrm{~b}$ wireless card which is installed in a builtin CompactFlash slot. The robots are equipped with high

\footnotetext{
${ }^{2}$ http://www.k-team.com/
} 


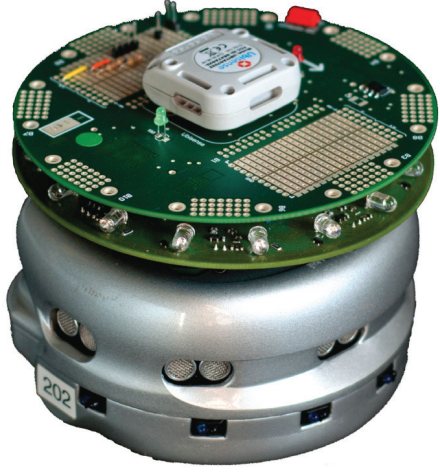

Fig. 7. The Khepera III robot is equipped with an extension range and bearing module which utilizes sixteen evenly-spaced infrared Light Emitting Diodes (LEDs). On top of this board, we mounted an active marker (LED) tracking module which simultaneously carries the Ubisense tag.

resolution wheel encoders, and an extension range and bearing module [14], which provides the robots with relative range and bearing measures. The noise values were experimentally determined on our actual hardware setup $\left(\sigma_{r}=0.2 \cdot r_{m n}\right.$, and $\sigma_{\theta}=0.2 \mathrm{rad}$ ), with a maximum detection range of $3 \mathrm{~m}$. Figure 7 shows the robot equipped with the range and bearing module on the lower level, and the LED ground-truth position tracking module on the upper level. The Ubisense UWB emitter tag is attached on the tracking board (barcode-side up). The overhead camera system is installed in combination with the open source tracking software SwisTrack [8] ${ }^{3}$. We use a GigE color camera which has a standard resolution of $1032 \times 778$ pixels, and is mounted $2.5 \mathrm{~m}$ above the robotic arena. The resulting picture resolution is 5.5 pixels per $\mathrm{cm}^{2}$, and the maximum error of the resulting ground truth positioning is below $3 \mathrm{~cm}$ (as reported in [13]).

The robot arena is delimited by a $30 \mathrm{~cm}$ high wall, but otherwise contains no obstacles (other than the robots themselves, which can occlude and thus prohibit relative range and bearing measurements). At the start of each experiment, the robots are randomly placed in the arena and have no knowledge of their pose (i.e., all particles are uniformly distributed over the

${ }^{3}$ http://en.wikibooks.org/wiki/SwisTrack

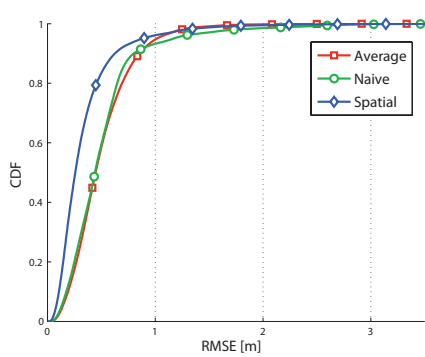

(a)

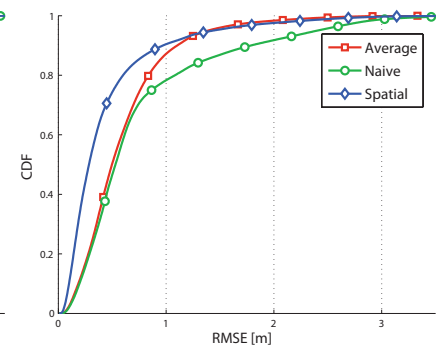

(b)
Fig. 8. Empirical cumulative density function of the localization errors over all runs, (a) for a collaborative multi-robot system, (b) for a non-collaborative system. The curves show results for the Average, Naive, and Spatial algorithm configurations. arena). For all experiments, the robots move straight at a speed of one robot-size per second $(12 \mathrm{~cm} / \mathrm{s})$ and avoid collisions. Relative range and bearing measurements as well as odometry updates are made at a frequency of $5 \mathrm{~Hz}$, the UWB tags emit positioning pulses at an update frequency of $1 \mathrm{~Hz}$, and the overhead camera tracks the robots at $10 \mathrm{~Hz}$. We perform 7 experiments (on our real setup) of $3 \mathrm{~min} 40 \mathrm{~s}$ each, and collect synchronized data sets consisting of unfiltered UWB TDOA measurements, relative robot range and bearing measurements, robot odometry measurements, and ground truth positioning information from the overhead camera. In order to evaluate the single-robot and multi-robot approaches separately, we divide our experimental configurations into non-collaborative and collaborative subcategories:

Non-collaborative 4 non-collaborative robots with only UWB TDOA data and odometry.

Collaborative 4 collaborative robots with relative observation data, UWB TDOA data, and odometry.

Then, in order to analyze the impact of information on the LOS/NLOS path condition $\mathrm{P}_{L_{u n}}$, we combine the above categories with each of the configurations listed below:

Naive No NLOS paths are assumed and $\mathrm{P}_{L_{u n}}=1, \forall u, \mathbf{x}_{n}$; this constitutes a naive approach in a mixed LOS/NLOS scenario.

Average The LOS proportion $\mathrm{P}_{L_{u n}}$ is as estimated by the system identification procedure; this corresponds to an average value valid over the arena as a whole (values can be looked up in Table I).

Spatial According to the algorithm shown in Section III-D, quasi-optimal spatial knowledge of the LOS/NLOS condition is available; $\mathrm{P}_{L_{u n}}=1$ when the estimated bias value $b_{u, n}$ is below some threshold (here $0.2 \mathrm{~m}$ ), for each individual position $\mathbf{x}_{n, t}$ where a TDOA measurement $\hat{\tau}_{u v, n, t}$ is received.

Each robot runs its localization algorithm with a set of 500 particles. We subsequently test the algorithm performance over 100 iterations for each dataset, for each of the 6 configurations elaborated above. We discuss the localization performance in terms of the mean positioning error of all particles in a given robot's belief (RMSE), a metric which implicitly includes the spread (or variance) of the particle positions. Figure 8 shows the empirical cumulative density function of the RMSE distribution over all runs, in (a) for the collaborative algorithm, and in (b) for the non-collaborative version. Table II reports the error values.

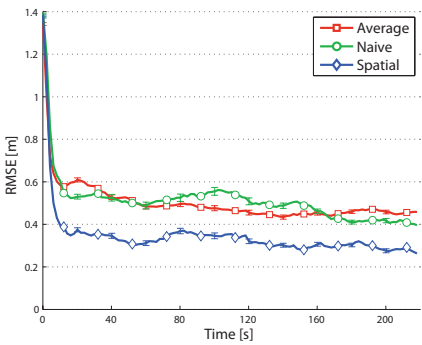

(a)

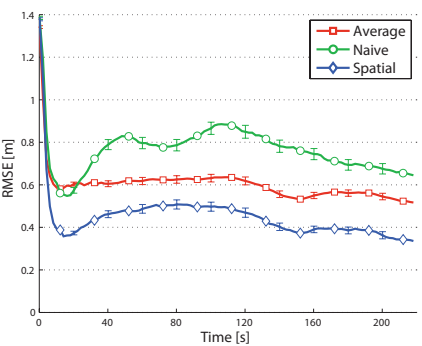

(b)
Fig. 9. Localization error over time, averaged over all runs (a) for a collaborative multi-robot system, (b) for a non-collaborative system. The curves show results for the Average, Naive, and Spatial algorithm configurations. The errorbars show a $95 \%$ confidence interval. 


\begin{tabular}{|c||c|c|c||c|c|c|}
\hline \multicolumn{1}{|c||}{} & \multicolumn{3}{c||}{ Collaborative } & \multicolumn{3}{c|}{ Non-collaborative } \\
\hline $\mathrm{P}(X \leq x)$ & Spatial & Average & Naive & Spatial & Average & Naive \\
\hline \hline $68.3 \%$ & $\mathbf{0 . 3 5}$ & 0.57 & 0.56 & $\mathbf{0 . 4 2}$ & 0.67 & 0.71 \\
\hline $95.5 \%$ & $\mathbf{0 . 9 2}$ & 1.04 & 1.21 & 1.52 & $\mathbf{1 . 4 4}$ & 2.46 \\
\hline $99.7 \%$ & 2.37 & $\mathbf{1 . 8 3}$ & 2.83 & 3.01 & $\mathbf{2 . 7 1}$ & 3.54 \\
\hline
\end{tabular}

TABLE II

ERRORS IN METERS FOR RESULTS SHOWN IN FIGURE 8

We observe that in general, the collaborative algorithm produces smaller localization errors than the non-collaborative algorithm. For instance, in the Spatial configuration for $\mathrm{P}_{L_{u n}}$, $95.5 \%$ of the time the error of the collaborative system is below $0.92 \mathrm{~m}$, in comparison to an error of $1.52 \mathrm{~m}$ respectively for the non-collaborative system. Indeed, by imposing additional geometric constraints through the relative observations, the collaborative robot team is more likely to converge to correct position estimates. Also, for any robot that has an approximate estimate of its true position, the propagation of this belief to its team-members will accelerate the process of localizing the whole system. Furthermore, we observe that our Spatial configuration will generally produce better results than the Average or Naive configurations, but is outperformed by the Average configuration for high cumulative densities. This is due to our approximative estimation of the bias values as explained in Section III-D, and thus in the worst case (i.e. when all base-stations are in NLOS), can lead to large outliers. Nevertheless, as is observed in Figure 9, the average localization error is lowest (below $0.4 \mathrm{~m}$ ) for the Spatial configuration, which is additionally confirmed by the very small confidence intervals. Finally, we observe that the collaborative system is able to compensate for the lack of NLOS knowledge of the Naive configuration with respect to the Average configuration, whereas in the non-collaborative system the performance of the Naive configuration deteriorates. Indeed, it is logical that when one can distinguish between LOS/NLOS conditions and estimate the NLOS bias (or where there are no NLOS situations), UWB alone is able to achieve good accuracy. Hence, the contribution of the collaborative system is especially important when LOS/NLOS conditions are unknown (which is the typical case). Finally, we note that although we used only a very modest particle set size (500 particles), the algorithm was able to achieve localization, and that for larger particle set sizes, an improvement on the localization accuracy is to be expected for all scenarios.

\section{CONCLUSION}

In this work, we considered the problem of localization for unknown initial conditions in NLOS scenarios. We presented a novel, probabilistic UWB TDOA error model, and based on data gathered on our real hardware setup, we estimated optimal model parameters. This error model was then introduced into a particle filter based localization algorithm, and validated in combination with on-board dead-reckoning information (odometry) in an experiment using multiple mobile robots. Additionally, we extended our particle filter algorithm to a fully scalable, decentralized adaptation enabling inter-robot collaboration. The results showed how our TDOA error model is able to improve localization performance by taking into account explicit knowledge of the LOS/NLOS path condition. Also, additional experiments showed how collaboration, through skillful exchange of positioning information in a multi-robot team, leads to a clearly improved localization performance. Further work will consider the estimation of the UWB error model parameters in real-time.

\section{REFERENCES}

[1] G. Benet, F. Blanes, J. E. Simó, and P. Pérez. Using infrared sensors for distance measurement in mobile robots. Robotics and Autonomous Systems, 40(4):255-266, 2002.

[2] M. Betke and L. Gurvits. Mobile robot localization using landmarks. Robotics and Automation, IEEE Transactions on, 13(2):251-263, 1997.

[3] D. Fox, W. Burgard, H. Kruppa, and S. Thrun. A probabilistic approach to collaborative multi-robot localization. Autonomous Robots, 8:325344, 2000.

[4] J. González, J. Blanco, C. Galindo, A. O. de Galisteo, J. FernándezMadrigal, F. Moreno, and J. Martínez. Mobile robot localization based on ultra-wide-band ranging: A particle filter approach. Robotics and Autonomous Systems, 57(5):496 - 507, 2009.

[5] D. Jourdan, J. Deyst, J.J., M. Win, and N. Roy. Monte carlo localization in dense multipath environments using uwb ranging. In IEEE International Conference on Ultra-Wideband (ICU), pages 314-319, 2005.

[6] J. J. Leonard and H. F. Durrant-Whyte. Directed Sonar Sensing for Mobile Robot Navigation. Kluwer Academic Publishers, 1992.

[7] H. Liu, H. Darabi, P. Banerjee, and J. Liu. Survey of wireless indoor positioning techniques and systems. IEEE Transactions on Systems, Man and Cybernetics, 37(6):1067-1080, 2007.

[8] T. Lochmatter, P. Roduit, C. Cianci, N. Correll, J. Jacot, and A. Martinoli. SwisTrack - A Flexible Open Source Tracking Software for MultiAgent Systems. In Proceedings of the 2008 IEEE/RSJ International Conference on Intelligent Robots and Systems, pages 4004-4010, 2008.

[9] A. I. Mourikis and S. I. Roumeliotis. Performance analysis of multirobot cooperative localization. IEEE Transactions on Robotics, 22(4):666681, 2006.

[10] K. P. N. Alsindi, B. Alavi. Measurement and modeling of ultrawideband TOA-based ranging in indoor multipath environments. IEEE Transactions on Vehicular Technology, 58:1046-1058, 2009.

[11] B. Neal. Slice sampling. The Annals of Statistics, 31:705-757, 2003.

[12] A. Prorok and A. Martinoli. A reciprocal sampling algorithm for lightweight distributed multi-robot localization. In Proceedings of the 2011 IEEE/RSJ International Conference on Intelligent Robots and Systems (IROS), 2011, To appear.

[13] A. Prorok, A. Arfire, A. Bahr, J. Farserotu, and A. Martinoli. Indoor navigation research with the Khepera III mobile robot: An experimental baseline with a case-study on ultra-wideband positioning. In International Conference on Indoor Positioning and Indoor Navigation (IPIN), 2010. doi: 10.1109/IPIN.2010.5647880.

[14] J. Pugh, X. Raemy, C. Favre, R. Falconi, and A. Martinoli. A fast on-board relative positioning module for multi-robot systems. IEEE Transactions on Mechatronics, 14(2):151-162, 2009.

[15] Y. Qi. Wireless geolocation in a non-line-of-sight environment. $\mathrm{PhD}$ thesis, Princeton University, 2004.

[16] Z. Sahinoglu, S. Gezici, and I. Guvenc. Ultra-wideband Positioning Systems. Theoretical Limits, Ranging Algorithms, and Protocols. Cambridge University Press, 2008.

[17] M. Segura, H. Hashemi, C. Sisterna, and V. Mut. Experimental demonstration of self-localized ultra wideband indoor mobile robot navigation system. In International Conference on Indoor Positioning and Indoor Navigation (IPIN), 2010. doi: 10.1109/IPIN.2010.5647457.

[18] S. Thrun, M. Bennewitz, W. Burgard, A. Cremers, F. Dellaert, D. Fox, D. Hahnel, C. Rosenberg, N. Roy, J. Schulte, and D. Schulz. Minerva: a second-generation museum tour-guide robot. In Proceedings of the 1999 IEEE International Conference on Robotics and Automation, volume 3, pages 1999-2005, 1999.

[19] S. Thrun, W. Burgard, and D. Fox. A real-time algorithm for mobile robot mapping with applications to multi-robot and $3 \mathrm{~d}$ mapping. In Proceedings of the IEEE International Conference on Robotics and Automation (ICRA), volume 1, pages 321-328, 2000.

[20] S. Thrun, W. Burgard, and D. Fox. Probabilistic Robotics. The MIT Press, 2005. 